\title{
Extreme genetic diversity in the type VII secretion system of Listeria monocytogenes suggests a role in bacterial antagonism
}

\author{
Kieran Bowran and Tracy Palmer*
}

\begin{abstract}
The type VII protein secretion system (T7SS) has been characterized in members of the phyla Actinobacteria and Firmicutes. In mycobacteria the T7SS is intimately linked with pathogenesis and intracellular survival, while in Firmicutes there is mounting evidence that the system plays a key role in interbacterial competition. A conserved membrane-bound ATPase protein, termed EssC in Staphylococcus aureus, is a critical component of the T7SS and is the primary receptor for substrate proteins. Genetic diversity in the essC gene of $S$. aureus has previously been reported, resulting in four protein variants that are linked to specific subsets of substrates. Here we have analysed the genetic diversity of the T7SS-encoding genes and substrate proteins across Listeria monocytogenes genome sequences. We find that there are seven EssC variants across the species that differ in their C-terminal region; each variant is correlated with a distinct subset of genes for likely substrate and accessory proteins. EssC1 is most common and is exclusively linked with polymorphic toxins harbouring a YeeF domain, whereas EssC5, EssC6 and EssC7 variants all code for an LXG domain protein adjacent to essC. Some essC1 variant strains encode an additional, truncated essC at their T7 gene cluster. The truncated EssC, comprising only the C-terminal half of the protein, matches the sequence of either EssC2, EssC3 or EssC4. In each case the truncated gene directly precedes a cluster of substrate/accessory protein genes acquired from the corresponding strain. Across $L$. monocytogenes strains we identified 40 LXG domain proteins, most of which are encoded at conserved genomic loci. These loci also harbour genes encoding immunity proteins and sometimes additional toxin fragments. Collectively our findings strongly suggest that the T7SS plays an important role in bacterial antagonism in this species.
\end{abstract}

\section{INTRODUCTION}

Protein secretion systems are found in almost all bacteria and are critical for processes such as nutrient capture and niche adaptation. To date, 10 distinct protein secretion systems (named types I-X) have been identified [1-3]. While most of these are exclusive to Gram-negative bacteria, the type VII secretion system (T7SS) is encoded by many Grampositive Actinobacteria and Firmicutes $[4,5]$. The T7SS has been heavily studied in pathogenic mycobacteria, where it is essential for virulence [6-8]. It is also linked to virulence in Staphylococcus aureus, supporting abscess formation during persistent infection $[9,10]$.

The T7SS comprises two subtypes - the T7a found in Actinobacteria and T7b in Firmicutes, that although related, have distinct differences $[11,12]$. A hexameric membranebound ATPase, termed EccC in Actinobacteria and EssC in Firmicutes, is common to both systems and is a critical component of the T7SS $[13,14]$. It lies at the centre of a multisubunit membrane complex that mediates protein transport across the cytoplasmic membrane [15-17]. The protein has four ATP-binding domains in its cytoplasmic C-terminus, each of which is essential for secretion activity [13, 14, 16-19], and the most C-terminal of these domains also plays a role in substrate recognition $[13,20,21]$. Substrates are recognized through a short C-terminal signal sequence, and secretion appears to be post-translational because at least some substrates are exported in a folded form [22-24].

In Firmicutes three additional membrane proteins, EsaA, EssA and EssB, and the cytoplasmic protein EsaB function alongside EssC to mediate T7 secretion [9, 25-29] (Fig. 1). Characterized substrates of the T7b system include proteins from the WXG and LXG families [9, 24, 30, 31]. In all T7b

Received 01 October 2020; Accepted 26 January 2021; Published 18 February 2021

Author affiliations: ${ }^{1}$ Microbes in Health and Disease Theme, Newcastle University Biosciences Institute, Newcastle University, Newcastle upon Tyne, NE2 $4 \mathrm{HH}, \mathrm{UK}$.

*Correspondence: Tracy Palmer, tracy.palmer@newcastle.ac.uk

Keywords: Listeria monocytogenes; type VII secretion; toxin; immunity protein; bacterial antagonism.

Abbreviations: CC, clonal complex; ST, sequence type; Tm, transmembrane helix; T7SS, Type VII secretion system.

Eight supplementary figures and three supplementary files are available with the online version of this article. 001034 @ 2021 The Authors 
(a)

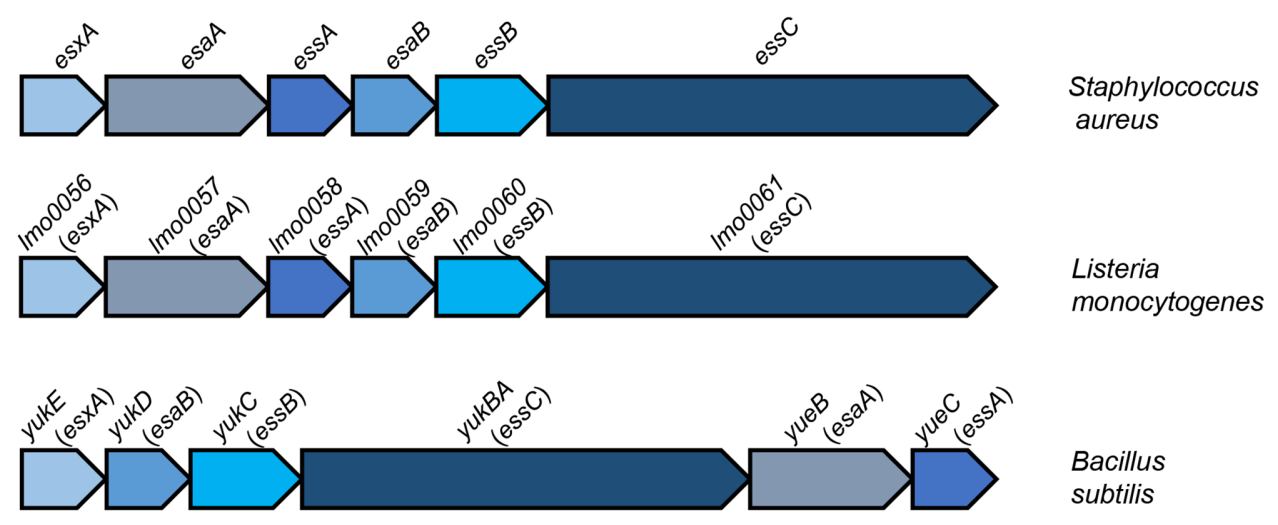

(b)

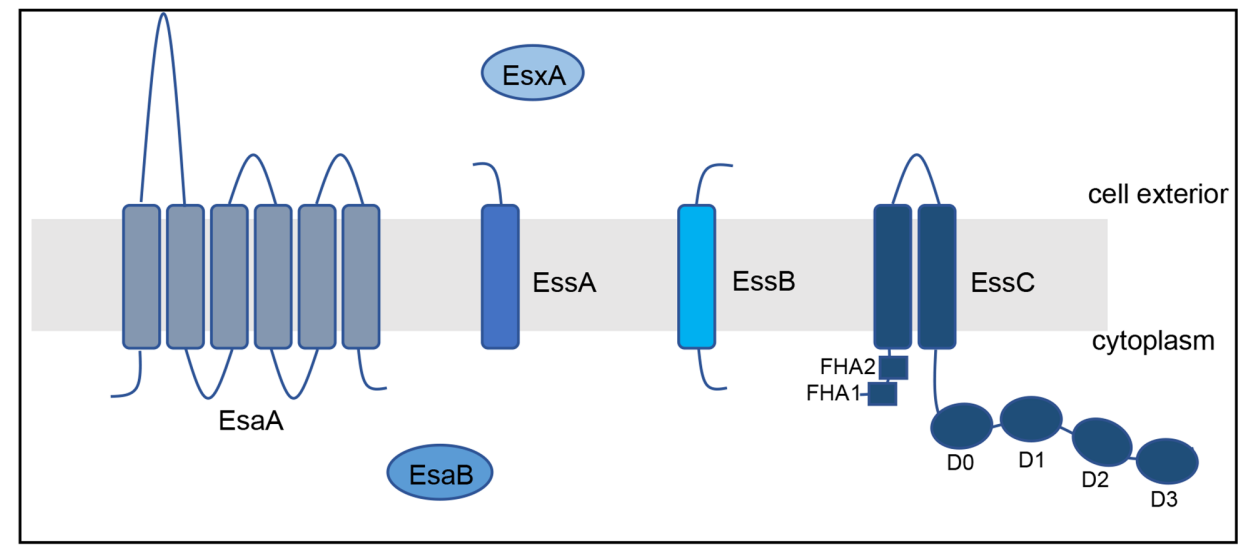

(c)

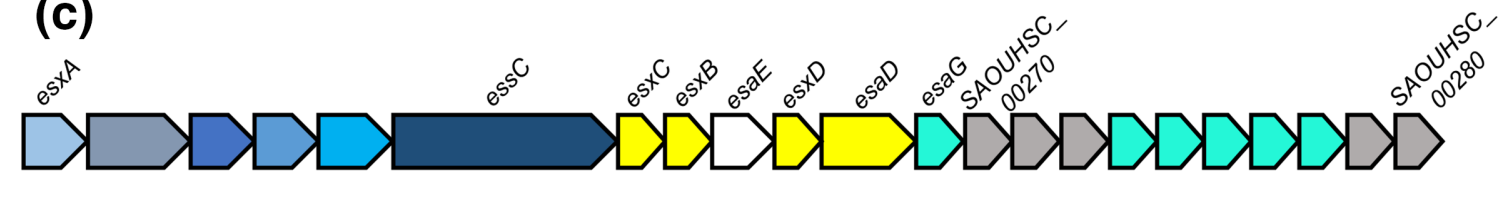

(d)

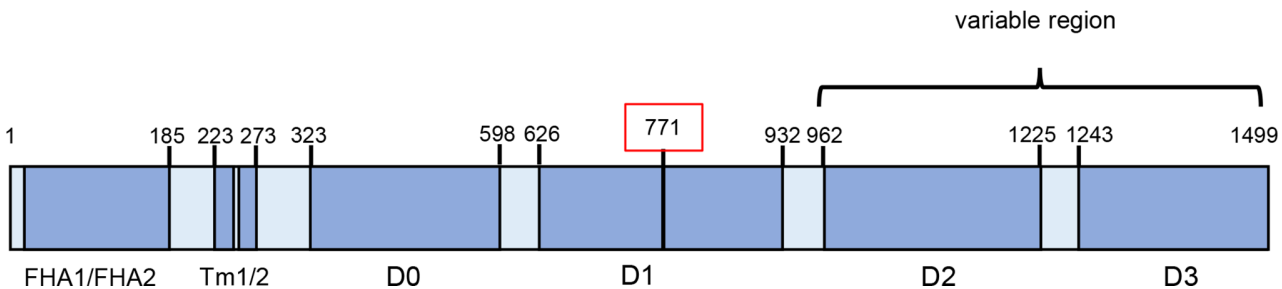

Fig. 1. The Firmicutes T7b secretion system. (a) Loci encoding the functional components of the T7b secretion system in Staphylococcus aureus, Listeria monocytogenes and Bacillus subtilis. The gene numbers for L. monocytogenes relate to the reference strain EGDe. (b) Subcellular location and topology of the T7b components. The two forkhead-associated domains (FHA1/FHA2) and the four ATPase domains (D0, D1, D2 and D3) of EssC are indicated. (c) The ess (T7) gene cluster of S. aureus strain NCTC8325. Genes encoding functional components are shaded in blue, secreted substrates in yellow, a chaperone protein in white and EsaG family immunity proteins in turquoise. (d) Schematic representation of L. monocytogenes EssC. Amino acid positions of the predicted domains are shown and the region that is variable across strains is indicated. Amino acid 771, shown boxed in red, is the position at which the truncated EssC proteins encoded in some L. monocytogenes essC1 strains start to align with the full-length protein. 
systems a conserved WXG protein, EsxA, is present. In S. aureus EsxA is essential for T7 secretion activity and may act as a structural/functional component of the secretion system $[9,25]$. The genes encoding these essential T7 components are arranged contiguously (Fig. 1), and are often found in gene clusters encoding secreted substrates and accessory proteins $[9,29,32]$.

The S. aureus T7SS is encoded at the ess locus. Although part of the core genome, it was noted that there is significant genetic diversity at the ess region across $S$. aureus strains [33]. Sequence divergence occurs part way through $e s s C$, giving rise to four EssC variants that differ in their $\mathrm{C}$-terminal domains. Each $\operatorname{ess} C$ variant is associated with a specific set of genes encoding T7 substrate proteins, and the variable C-terminal domain of EssC has been implicated in the recognition of strain-specific substrates [21,33].

The most commonly studied S. aureus strains encode the EssC1 variant (Fig. 1c). These strains produce a T7-secreted nuclease, EsaD, a member of the YeeF protein family, and are protected from self-intoxication by an immunity protein, EsaG $[34,35]$. A chaperone protein, EsaE, interacts with both $\mathrm{EsaD}$ and EssC and likely targets the nuclease to the secretion machinery [34, 36]. Strains harbouring ess C1 account for approximately $50 \%$ of sequenced S. aureus [33]. Analysis of EsaD sequences across these strains shows that the nuclease domain is polymorphic, suggestive of an anti-bacterial toxin [34]. All S. aureus strains, including essC2, ess C3 and essC4 strains that do not encode EsaD, have multiple genes coding for EsaG homologues at their ess loci, suggesting that EsaD targets staphylococci (Fig. 1c) [33, 34]. An anti-staphylococcal role for EsaD has been confirmed in competition experiments, where strains were sensitized to killing by the nuclease when esa $G$ genes were deleted $[34,37]$.

Since the identification of EsaD, a growing number of T7-secreted antibacterial toxins have been described. TspA, a second polymorphic toxin found in S. aureus, has membranedepolarizing activity that is neutralized by the TsaI immunity protein [37]. Streptococcus intermedius produces at least three antibacterial toxins, of which TelB is an NADase and TelC a lipid II phosphatase [30]. Moreover, recent reports indicate that the T7b systems of Bacillus subtilis and Enterococcus faecalis also have antibacterial activity $[38,39]$, indicating that bacterial antagonism is a common feature of several Firmicutes T7SSs.

Listeria monocytogenes is a Gram-positive firmicute and human foodborne pathogen that is able to invade and replicate intracellularly [40]. A T7SS is encoded in the genome and has been partially characterized in strains EGDe and 10403s, where it was shown to be dispensable for virulence and host cell invasion [32, 41]. Here we have analysed the genetic diversity at the T7 gene clusters of sequenced L. monocytogenes strains. We show that seven variants of EssC, which differ in their C-terminal region, are encoded across L. monocytogenes. Each variant is associated with specific genes encoding likely substrate proteins. We identify polymorphic toxins that fall into the YeeF (EsaD) and LXG families and clusters of immunity proteins for self and nonself toxins. Our observations point to a major role for the T7SS in interbacterial competition.

\section{METHODS}

L. monocytogenes EGDe serovar 1/2a (NC_003210.1) was used as the reference strain in this analysis. EssC, encoded by $l m o 0061$, was used as the query for Basic Local Alignment Search Tool Protein (BLASTP) analysis using the National Center for Biotechnology Information's (NCBI's) Reference Sequence (RefSeq) database of non-redundant protein sequences [42]. All identified L. monocytogenes EssC homologues were assigned a percentage identity to Lmo0061 using the BLOSUM62 scoring matrix and an expect threshold of 10. EssC sequence variants were aligned using the CLUSTALW algorithm of the European Bioinformatic Institute (EBI) via the Clustal OMEGA software (v.1.2.4) [43], and protein sequence alignments were viewed in Jalview [v.2.11.1.0] [44]. EssC sequences were further analysed through the construction of maximum-likelihood phylogenetic trees using the MEGAX software package (v.10.1.8) [45, 46]. The Pasteur Institute's Listeriomics tool [47] was used to acquire sequences of EssCs across 60 annotated L. monocytogenes genomes with information on lineage, clonal clusters and serotypes. The presence of sigma 70 promoters and terminator regions within the T7b locus was evaluated using в вRом and FindTerm, respectively [48]. Candidate Rho-independent terminator regions were analysed using ARNold [49].

Protein accession numbers were subjected to flanking genes (FlaGs) analysis [50] to establish the conservation of $\mathrm{T} 7 \mathrm{~b}$ genes across multiple L. monocytogenes strains. Gene products were analysed using BLASTP analysis, and the presence of transmembrane regions was predicted using TMHMM [51]. Homology modelling was achieved using the Protein Homology/analogy Recognition Engine (PHYRE [52]).

\section{RESULTS}

\section{The T7SS gene cluster of $L$. monocytogenes}

The functional components of the L. monocytogenes T7SS are encoded contiguously from $\operatorname{lmo0056}$ (es $x A$ ) through to Imo0061 (essC; Fig. 1a; all gene numbers relate to type strain EGDe), with the gene order mirroring that of the $S$. aureus ess locus. In S. aureus, a peptidoglycan hydrolase, EssH, is encoded divergently to es $x A$ and is essential for T7 substrate secretion across the cell wall [53]. Analysis of the genomic region immediately upstream of es $x A$ in $L$. monocytogenes did not identify a homologue of ess $H$ at this locus, or elsewhere on the chromosome. A stem-loop structure is present in the $S$. aureus es $x A-e s a A$ intergenic region [25]. A similar stemloop with an estimated Gibbs free energy of $-13.6 \mathrm{kcal} \mathrm{mol}^{-1}$ and sharing $62 \%$ identity with that of S. aureus, is conserved across the sequenced $L$. monocytogenes genomes. In S. aureus the stem-loop plays a role in regulating the expression level of the genes immediately downstream of es $x A$, which are transcribed 10-100 fold lower than esxA $[25,54]$. It likely 
Table 1. Seven EssC variants identified encoded across L. monocytogenes genome sequences present in RefSeq

\begin{tabular}{|lcccc|}
\hline Variant & Sequence length (aa) & C-terminal amino acid sequence & No. of sequences & Percentage frequency \\
\hline EssC1 & 1498 & IAPNQ & 71 & 56 \\
EssC2 & 1498 & YEEEV & 56 & $23 \%$ \\
EssC3 & 1496 & KIPKG & 36 & $16 \%$ \\
EssC4 & 1501 & RMPKH & 33 & $14 \%$ \\
EssC5 & 1483 & IKLPN & 7 & $3 \%$ \\
EssC6 & 1500 & VKEEE & 12 & $5 \%$ \\
EssC7 & 1490 & VLSKS & $12 \%$ \\
\hline
\end{tabular}

has a similar role in L. monocytogenes, as it has been reported that ess $C$ and ess $B$ are much more weakly expressed than es $x A$ in strain EGDe [41].

\section{Seven distinct EssC variants are encoded across L. monocytogenes strains}

Analysis of the amino acid sequence of the T7 core components indicates that five of them (EsxA, EsaA, EsaB, EssA and EssB) have high sequence conservation (approximately $97 \%$ identity across strains present in RefSeq). By contrast, EssC sequences are much more variable, with less than $80 \%$ identity between strains. To further analyse the sequence variability of EssC, all L. monocytogenes full-length EssC sequences (274 in total) were extracted from NCBI RefSeq and aligned. It was observed that the proteins fall into seven different groups, here named EssC1-7. The sequences of EssC1 and EssC3 diverge from the other EssC groups at around the start of domain D2 (Figs 1a and S1, available in the online version of this article). The five other EssC variants are almost invariant in their proximal D2 sequences, starting to diverge from one another approximately 180 amino acids into this domain. As shown in Table 1, EssC1 is the most common variant, being encoded in $26 \%$ of sequenced L. monocytogenes strains, and EssC6 the least common, with only $3 \%$ occurrence.

To assess the distribution of ess $C$ variants across clonal complexes and evolutionary lineages $[55,56]$, the 65 complete L. monocytogenes EssC sequences were extracted from the Pasteur Institute's publicly available annotated collection and used to construct a maximum-likelihood phylogenetic tree (Fig. 2). While essC6 strains are notably absent from the phylogenetic tree, the six other EssC variants are represented. The tree divides into two main branches, with the first comprising EssC2, EssC4, EssC5 and EssC7, and the second solely comprising EssC1. As with the NCBI RefSeq database, the majority of the Pasteur Institute sequences encode EssC1 or EssC2, which both show distribution across evolutionary lineages I and II. The remaining ess $C$ variants are confined to a single lineage, with the exception of $L$. monocytogenes FSL J1-208, which is the only essC7 strain to belong to lineage IV out of those analysed. The four essC3 strains belonging to CC69 of lineage III cluster outside of the main two branches away from the other variants.

\section{Two variable genomic regions are found at the T7 gene cluster of $L$. monocytogenes}

It was noted for $S$. aureus that genetic diversity at the ess locus starts within the ess $C$ gene and continues downstream [33]. Inspection of the genomic region $3^{\prime}$ of $L$. monocytogenes ess $C$ reveals that it is exceptionally diverse across different strains, in agreement with prior studies that identified this region as a hypervariable hotspot $[57,58]$. We identified two closely spaced regions of high variability (Figs $3 a$ and S2). The first of these variable regions is bounded by ess $C$ at the $5^{\prime}$ side and at the $3^{\prime}$ side by a cluster of housekeeping genes (Imo0075lmot01; encoding a predicted phosphoenolpyruvate mutase, 6-O-methylguanine DNA methyltransferase, a protein of the YjbI superfamily, a predicted D-isomer-specific 2-hydroxyacid dehydrogenase and a tRNA, respectively). The second variable region lies between $\operatorname{lmot} 1$ at the $5^{\prime}$ end and $l$ mo0082 (encoding a hypothetical membrane protein) at the $3^{\prime}$ end (Fig. 3a).

\section{YeeF family toxins are encoded in variable region 1 of $L$. monocytogenes essC 1 strains}

Although variable region 1 is highly diverse across L. monocytogenes, strains with the same ess $C$ variant show some common features. For example, a shared set of genes are encoded immediately downstream of ess $C$ in all ess $C 1$ strains (Figs $3 \mathrm{~b}$ and S2). The first three of these genes, lmo0062, Imo0063 and $l m o 0064$, are syntenous with the three genes that are adjacent to ess $C 1$ in $S$. aureus strains (Fig. 1c), and encode homologues of the substrate proteins EsxC (Lmo0062) and EsxB (Lmo0063), and the chaperone protein EsaE (Lmo0064). Lmo0065 is also common to all essC1 strains and encodes a small helical protein with some limited similarity to $S$. aureus EsxD.

The ess $C 1$ sequences start to diverge at $1 m 00066$. In each ess $C 1$ strain this gene codes for a protein of the YeeF superfamily, to which the $S$. aureus toxin EsaD also belongs. However, we note that the encoded proteins have a highly variable C-terminal toxin domain across variant 1 strains. In total, 10 different toxin domains could be identified (Fig. S3, Table 2). In each case, the $\mathrm{N}$-terminal 400 amino acids (the 'YeeF domain') are highly conserved and this region is predicted to be almost completely a-helical. In S. aureus 


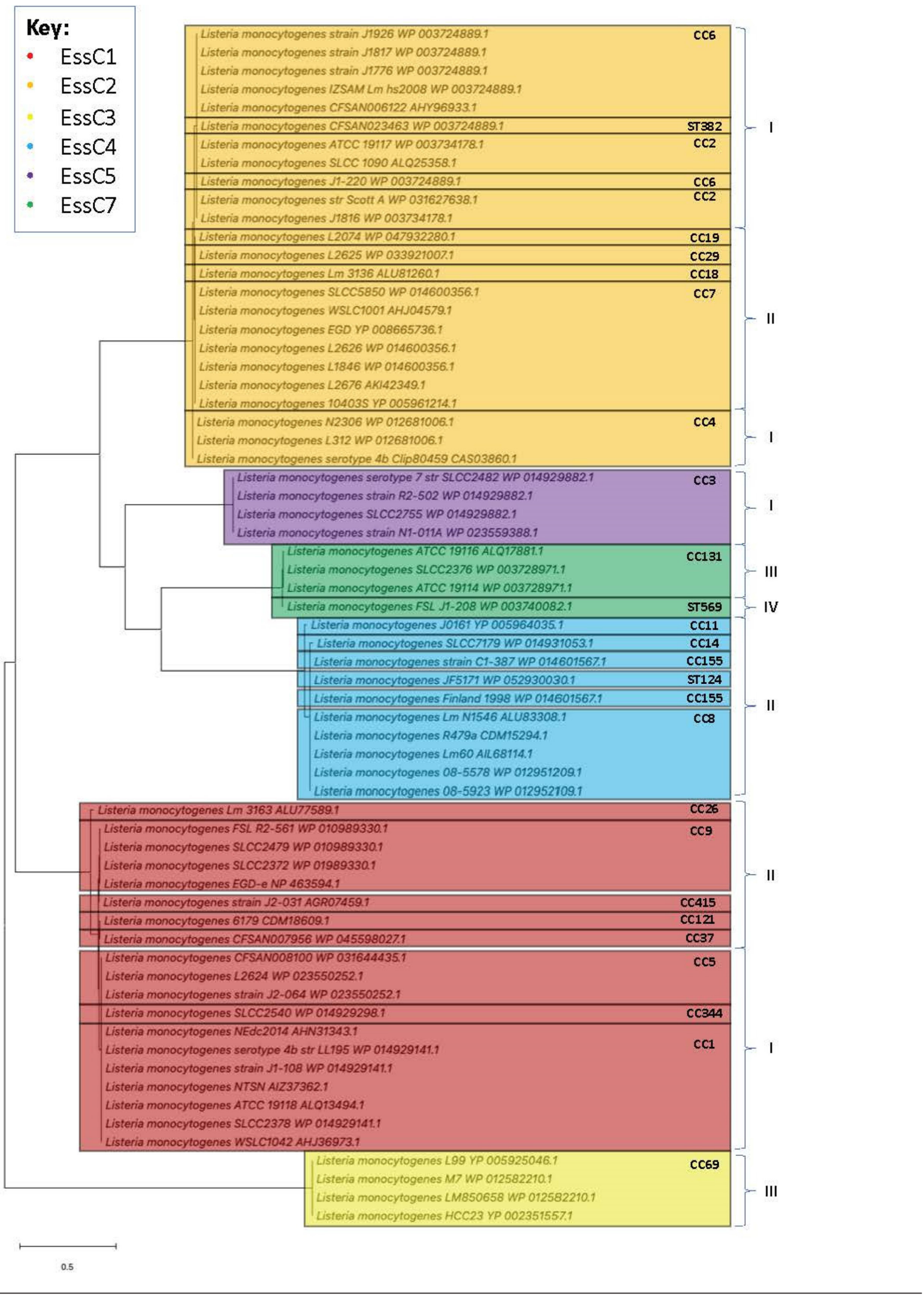

Fig. 2. Phylogenetic distribution of essC variants across strains of $L$. monocytogenes. Maximum-likelihood phylogenetic tree showing the presence of multiple essC variants across the four evolutionary lineages (I-IV) of L. monocytogenes. CC, clonal complex; ST, sequence type. The scale bar represents the number of substitutions per site. 
(a)

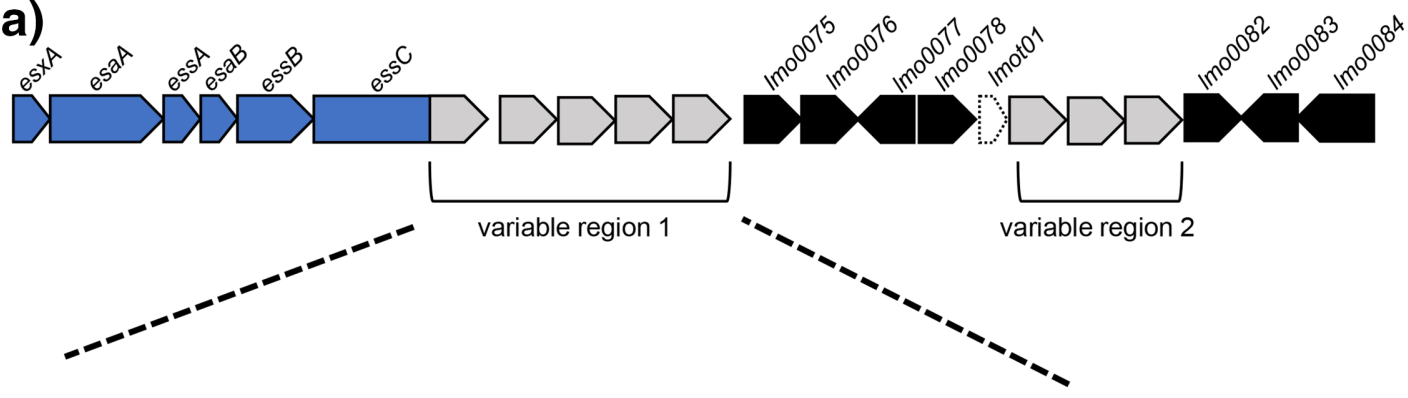

(b)
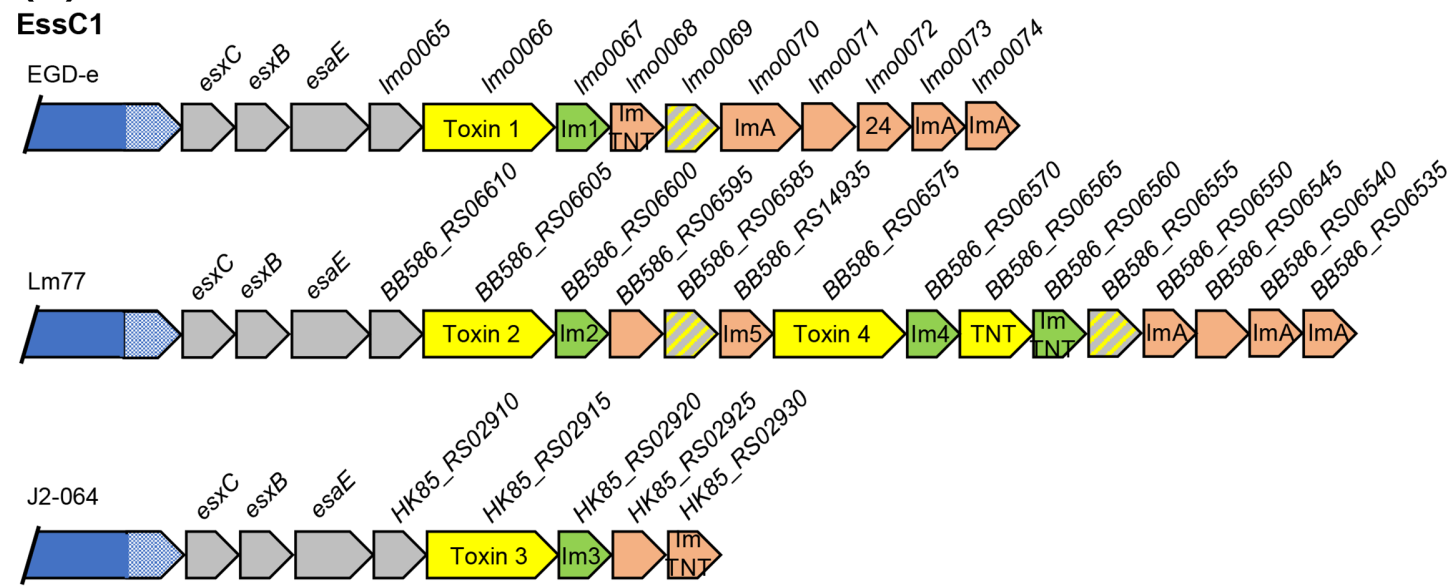

(c)

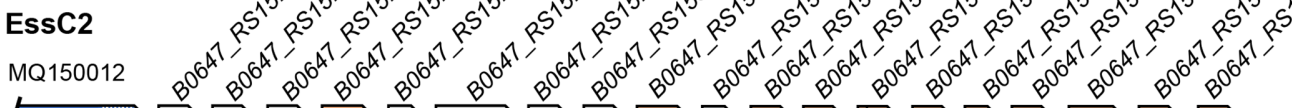

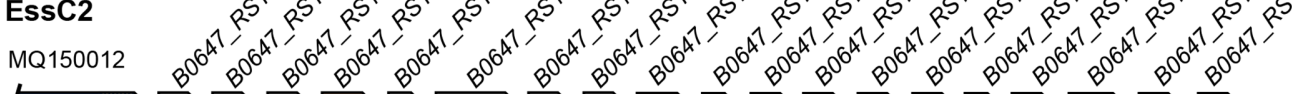

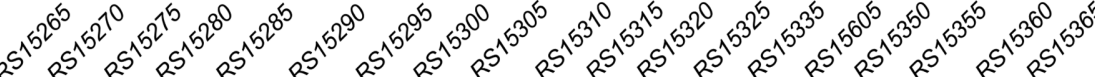

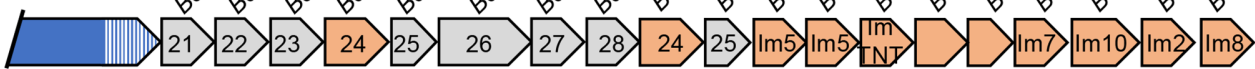

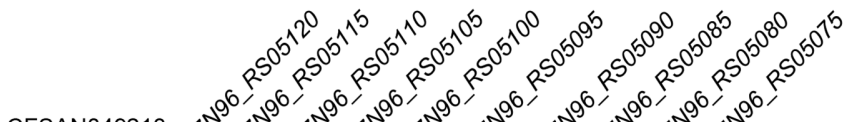

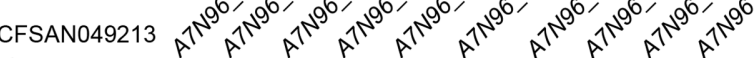

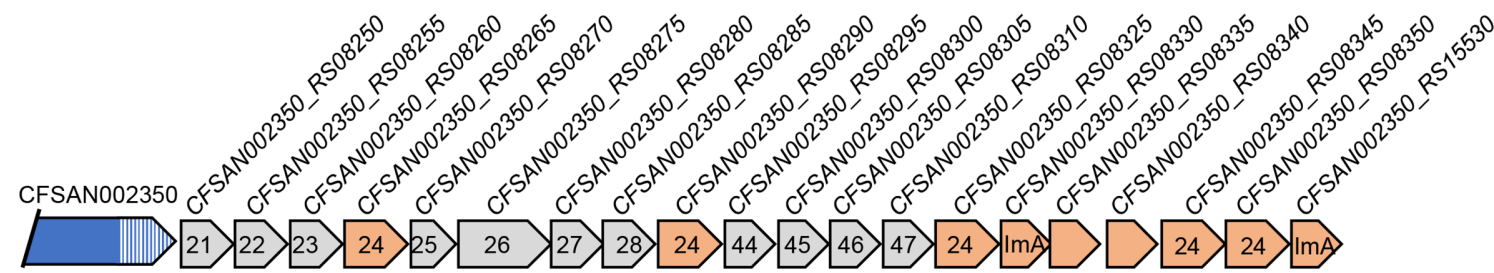

Fig. 3. Two genetically variable regions can be identified downstream of essC. (a) Schematic representation of the T7-encoding region on the chromosome of L. monocytogenes, highlighting the positions of two genetically variable regions. Genes shown in blue encode functional components of the T7SS and genes in black are invariant. The gene shown in dashed lines encodes a tRNA. (b, c) Three genetic arrangements of variable region 1 from (b) essC1 and (c) essC2 strains. Genes encoding candidate toxins are shaded yellow with the cognate immunity gene in green. Probable orphan immunity proteins are shown in peach (with likely immunity indicated where identified) and genes encoding toxin fragments in hatched grey and yellow shading. Genes numbered 21-28 are conserved in the same position across all essC2 strains. Gene 24 in this cluster is predicted to encode an immunity protein and is also found in the EGDe essC1 strain. Genes numbered 44-47 are common to essC 4 strains. Gene sizes and intergenic regions not to scale. 
Table 2. Ten distinct YeeF family toxin variants are encoded in variable region 1 of essC1 strains

\begin{tabular}{|c|c|c|c|}
\hline Toxin & Sequence length (aa) & Predicted function & Probable immunity \\
\hline Toxin 1 & 604 & VIP2 superfamily: ADP ribosyltransferase & Im1: ADP ribosyl glycohydrolase \\
\hline Toxin 2 & 557 & Unknown & $\operatorname{Im} 2$ : unknown \\
\hline Toxin 3 & 577 & Predicted ribonuclease & Im3: SUKH-1 family immunity protein \\
\hline Toxin 4 & 565 & Predicted ribonuclease & Im4: SUKH-1 family immunity protein \\
\hline Toxin 5 & 564 & Structurally related to Tne2: NADase? & $\begin{array}{c}\text { Im5: bipartite? haemagglutinin-related protein/Ssp } \\
\text { translocon-related protein }\end{array}$ \\
\hline Toxin 6 & 598 & Ntox15 superfamily: predicted ribonuclease & $\begin{array}{l}\text { Im6: DUF1851 domain-containing protein: } \\
\text { structurally related to type VI immunity protein } \\
\text { Atu4351 from Agrobacterium tumefaciens }\end{array}$ \\
\hline Toxin 7 & 563 & Structurally related to Tne2: NADase? & Im7: unknown \\
\hline Toxin 8 & 609 & $\begin{array}{l}\text { Structurally related to Streptococcus pyogenes Spn: } \mathrm{NAD}^{+} \\
\text {glycohydrolase? }\end{array}$ & Im8: unknown \\
\hline Toxin 9 & 556 & Unknown & Im9: unknown \\
\hline Toxin 10 & 578 & Unknown & Im 10: unknown \\
\hline
\end{tabular}

variant 1 strains, the EsaE chaperone recognizes the YeeF domain of EsaD, and it is likely that this highly conserved domain in L. monocytogenes essC1 toxins is also recognized by EsaE. The toxin domains are predicted to have functions such as ADP ribosyltransferase, ribonuclease or NADase activity (Table 2), and each toxin is always paired with the same candidate immunity protein encoded directly downstream. Some essC1 strains encode two YeeF domain proteins within this region, and some also encode a protein with a tuberculosis necrotizing toxin (TNT) domain, although this lacks a detectable YeeF domain (Figs $3 \mathrm{~b}$ and $\mathrm{S} 2$ ). Other genes found within this region of essC1 strains encode toxin fragments and orphan immunity proteins.

\section{A conserved set of genes are found downstream of essC2}

All essC2 strains have a common set of genes immediately downstream of ess C (Fig. 3c; numbered 21-28 for variant 2 genes 1-8). Most of these genes encode small proteins ( $>150$ amino acids) of unknown function. The largest protein encoded in this cluster, represented by B0647_RS15290 (gene 26 in Fig. 3c) is approximately 320 amino acids in size and is strongly predicted to be a D-isomer-specific 2-hydroxyacid dehydrogenase family protein. Curiously, this is the same predicted function as that for the gene encoded by $\operatorname{lmo0078}$ in the invariant housekeeping cluster, although the two proteins have no detectable sequence identity. Two of the genes in the ess $C 2$ variable region 1 cluster encode related proteins (24 in Fig. 3c). This duplication is seen in all strains, and there is always an approximate 500 bp intergenic region between the last two genes in the cluster.

Genes $24-28$ in this cluster seem to form a module of six genes (always in the order 24-25-26-27-28-24; with an extended intergenic region between genes 28 and 24). This module is often but not always found in variable region 1 of other ess $C$ variants, and sometimes additional copies of gene 24 also flank this cluster (Fig. S2). Within this module genes 24 and 25 may form a pair, as they are occasionally co-occur away from the other genes of the module. Many orphan copies of 24 are also found in the T7 gene clusters of most ess $C$ variants, suggesting that it may encode an immunity protein.

Downstream of these nine conserved genes the essC2 strains are much more variable, but many strains encode multiple predicted antitoxins, including to the YeeF and LXG domain toxins present in other L. monocytogenes strain variants.

\section{essC3 strains may encode a novel toxin}

Many essC3 strains analysed have a very short variable region 1, comprising two genes of unknown function (Fig. 4a). The second gene of the pair encodes a protein of approximately 660 amino acids, which is a similar size to other T7b toxins, although it is unrelated to either the LXG or YeeF domain proteins. It therefore may represent a novel toxin. A few essC3 strains have larger gene clusters in variable region 1 , with a few carrying the six-gene module $[24,25]$ that is present in all essC2 strains. Orphan immunity proteins to other T7b toxins are also found in this region in some strains.

\section{Seven conserved genes are found downstream of essC4}

The first seven genes downstream of ess $C 4$ are conserved in all ess C4 strains (41-47 in Fig. 4b). Most of the encoded proteins are $<200$ amino acids; the largest protein encoded by the cluster, at 433 amino acids, is the product of gene 44. Most of the proteins lack any identifiable domains, with the exception of 41 , which is a member of the SMC_prok_A 

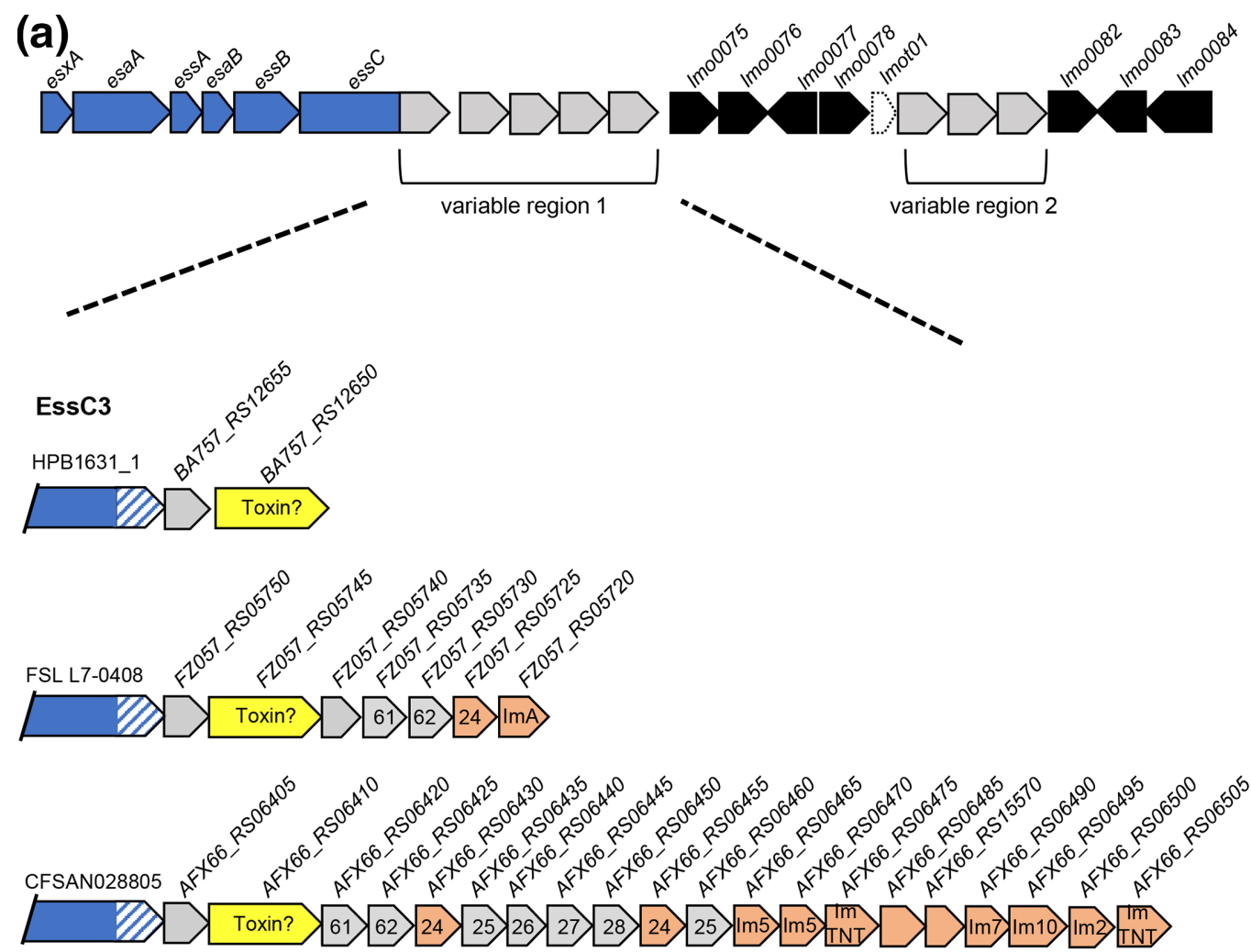

(b)
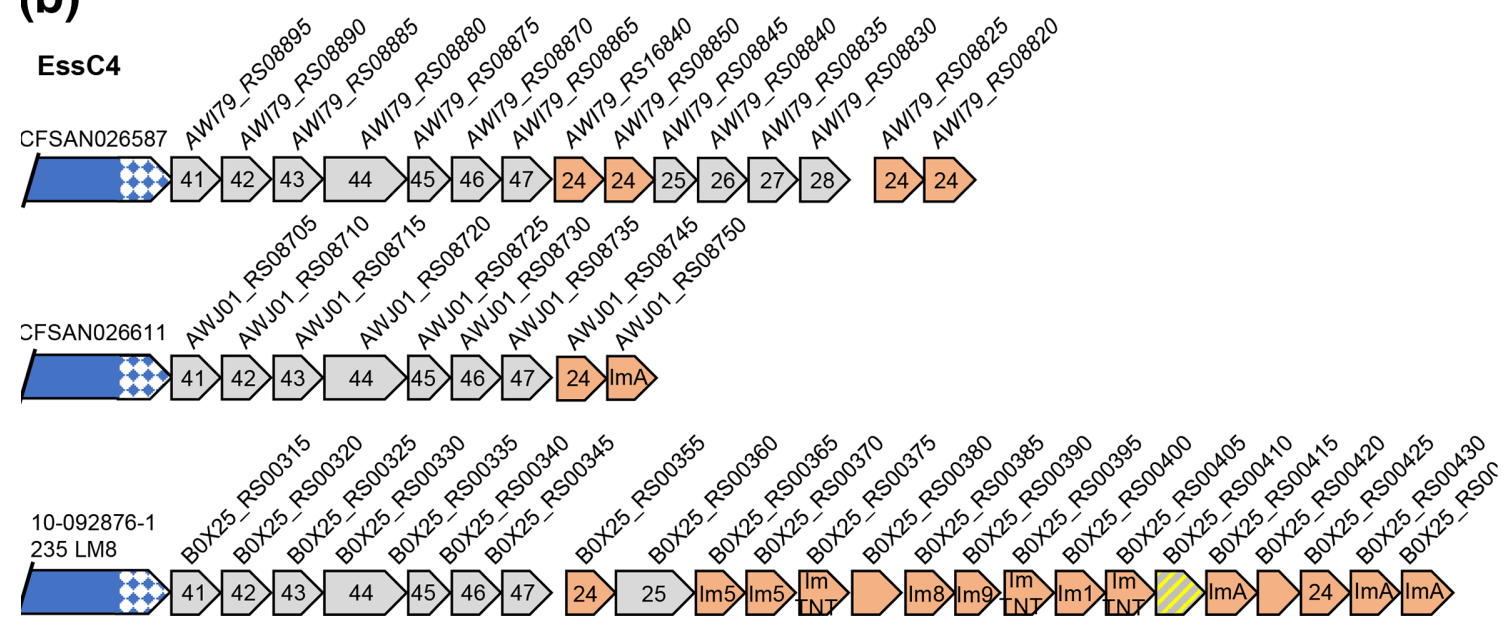

Fig. 4. Genetic variability of region 1 in essC3 and essC4 strains. (a, b) Three genetic arrangements of variable region 1 from (a) essC3 and (b) essC4 strains. Genes encoding candidate toxins are shaded yellow. Probable orphan immunity proteins are shown in peach (with likely immunity indicated where identified) and genes encoding toxin fragments in hatched grey and yellow shading. Genes numbered 41-47 are common across all essC4 strains. Genes numbered 24-28 are common to essC2 strains, and genes numbered 61 and 62 are common to essC6 strains. Gene sizes and intergenic regions not to scale.

superfamily, and the C-terminal 65 amino acids of 45 , which is an Mfa2 superfamily domain.

The last four genes in this cluster form a module - they are found in variable region 1 of other ess $C$ variant strains (e.g.
Figs $3 \mathrm{c}$ and S2). Interestingly, in this module gene 44 is shorter and encodes a protein of only 335 amino acids. The shorter product aligns with $100 \%$ sequence identity to the longer 44 protein from amino acid 99 onwards, but lacks the $\mathrm{N}$-terminal 

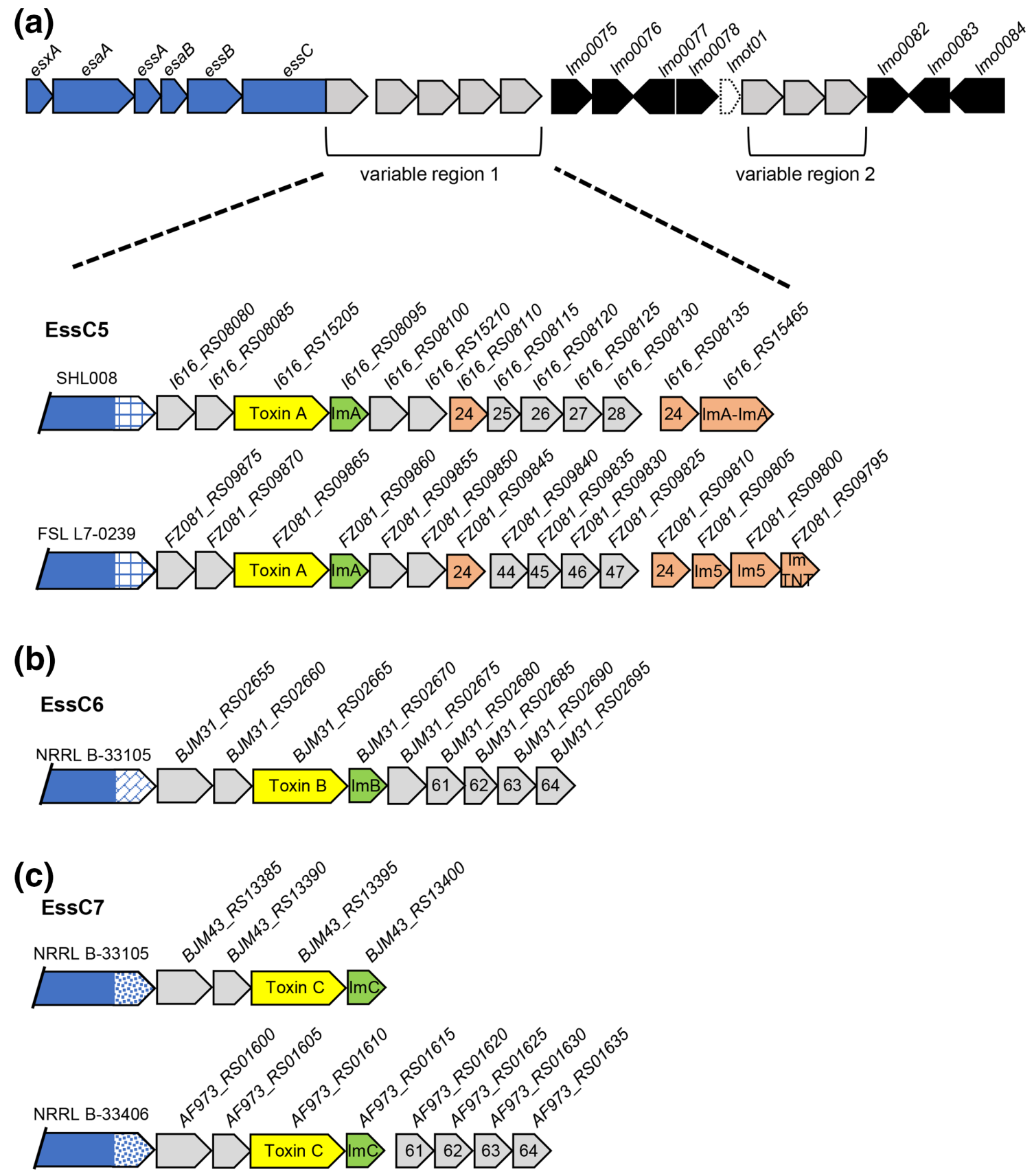

Fig. 5. An LXG toxin is encoded in variable region 1 of essC5, essC6 and essC7 strains. (a, c) Two genetic arrangements of variable region 1 from (a) essC5 and (c) essC7. (b) A single genetic arrangement is seen for essC6 strains. Genes encoding candidate toxins are shaded yellow with the cognate immunity gene in green. Probable orphan immunity proteins are shown in peach (with likely immunity indicated where identified). Genes numbered 61-64 are common to essC6 strains. Genes numbered 24-28 are common to essC2 strains and those numbered 44-47 are common to essC4 strains. Gene sizes and intergenic regions not to scale.

region. Several essC4 strains also encode a copy of the ess $C 2$ six-gene module at this locus (Fig. 4b).

\section{essC5, essC6 and essC7 strains encode an LXG toxin in variable region 1}

Variable region 1 of essC5, essC6 and essC7 strains shares some superficial similarity (Fig. 5). An LXG family toxin is always encoded by the third gene downstream of ess $C$, followed by a probable immunity protein. The three toxins (toxin $\mathrm{A}$ in ess C5 strains, toxin B in essC6 strains and toxin $\mathrm{C}$ in ess $\mathrm{C7}$ strains) share some limited sequence identity (approximately 15-20\%; Table 3, Fig. S4) in the first 270 amino acids, but are less similar in the C-terminal toxin domains. Nonetheless, all three proteins can be structurally modelled with 


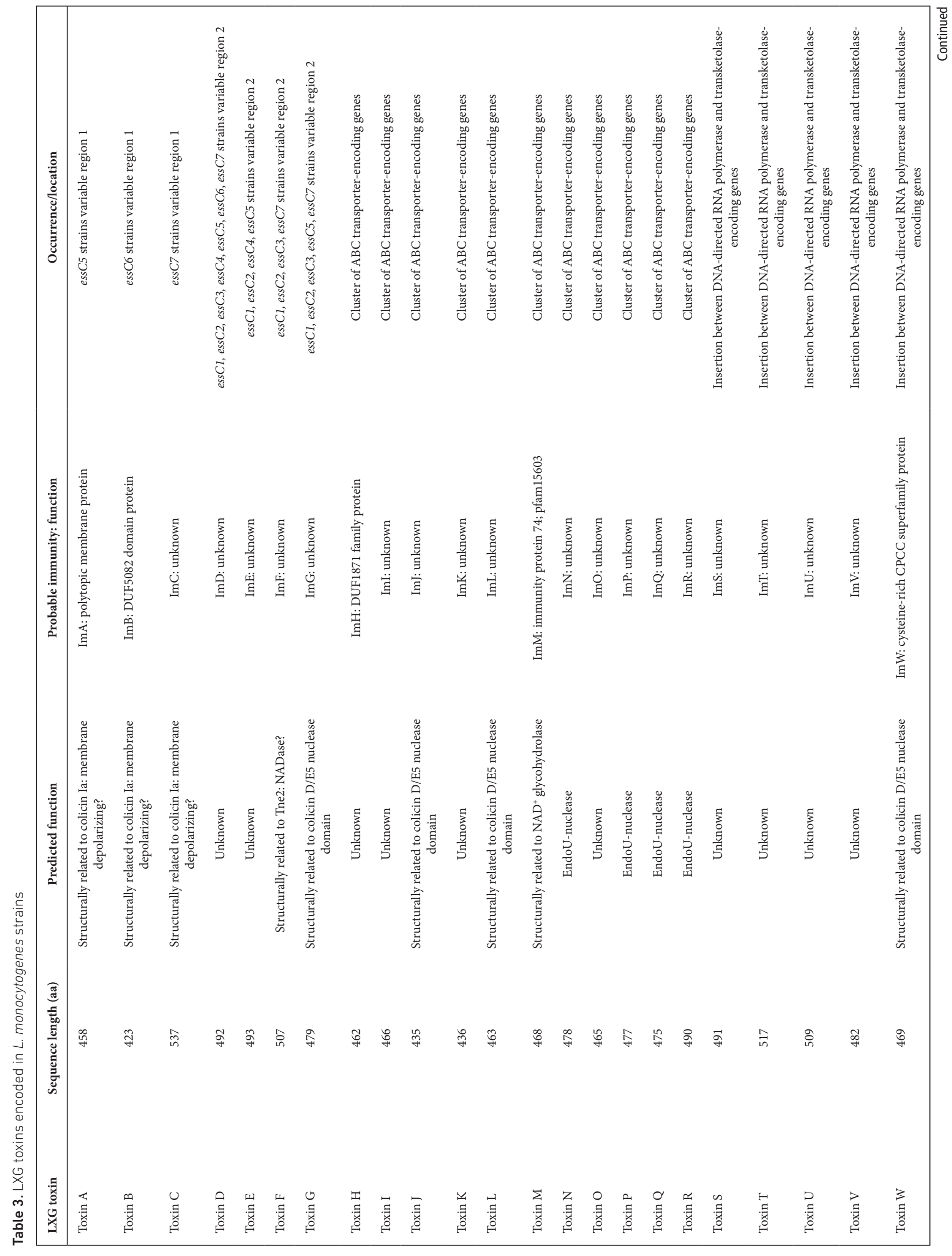




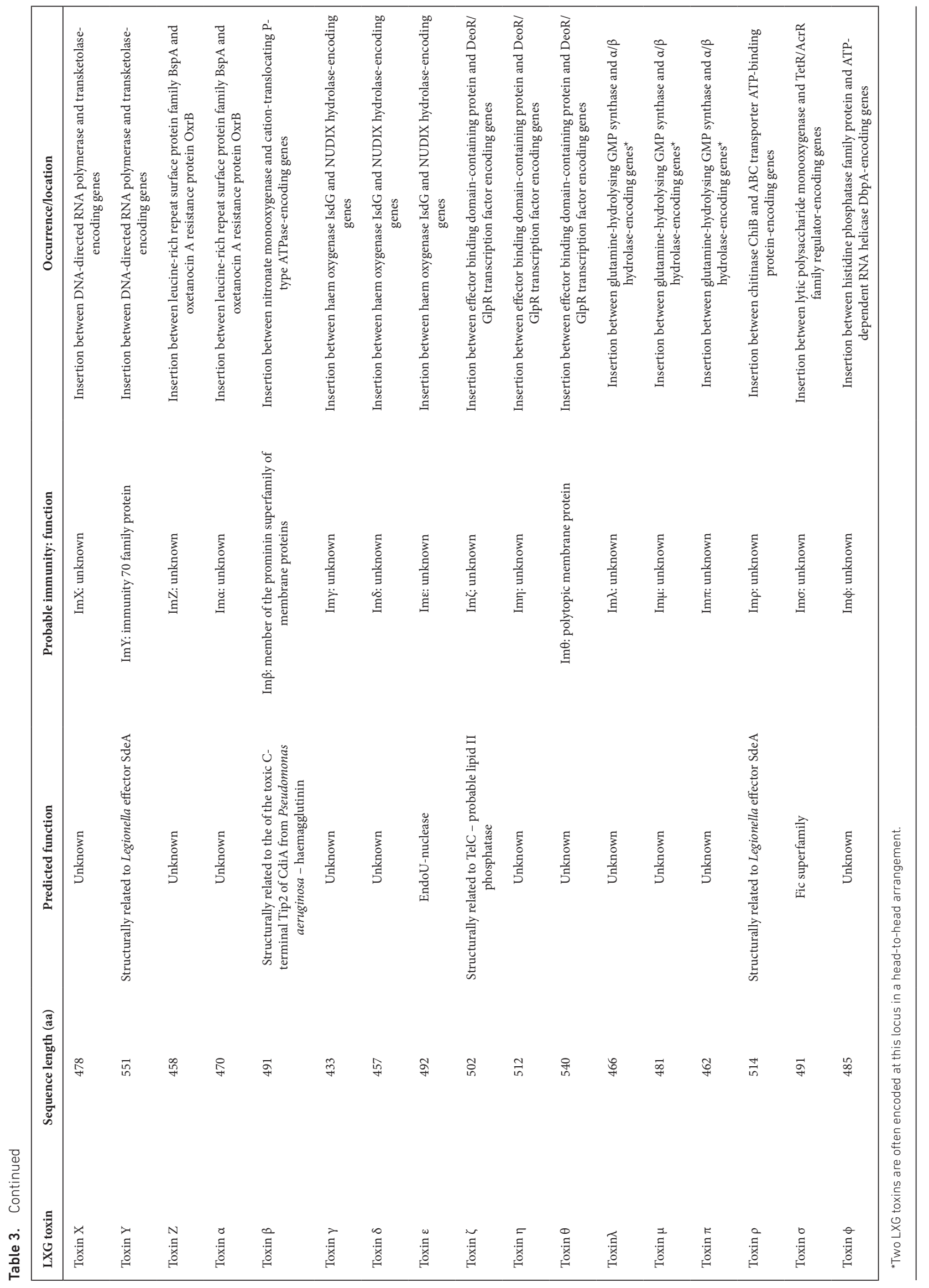


99\% confidence using colicin Ia as a template (PHYRE [52]). Recently it has been shown that TspA, a T7-secreted protein from $S$. aureus that can also be modelled on the colicin Ia, is a membrane-depolarizing toxin [37], suggesting that these three L. monocytogenes toxins may also have a similar mode of action. The candidate immunity proteins for these toxins fall into three unrelated protein families (Figs 5 and S2, Table 3).

The essC5 strains always encode a pair of small proteins of unknown function in-between essC5 and the toxin A gene. Downstream of the candidate immunity gene the ess C5 strains start to diverge (Fig. 5a), with some strains encoding the six-gene module found in ess $\mathrm{C} 2$ strains, while others have the five-gene module found in essC4.

All essC6 strains sequenced to date have an identical set of genes in variable region 1 (Fig. 5b). The first gene in this region encodes a 367 amino acid product that appears to be structurally related to a metallopeptidase (match to the crystal structure of the m16b metallopeptidase subunit from Sphingomonas sp. al with $96 \%$ confidence; HYRE [52]). The second gene encodes a 93 amino acid peptide that is a member of the DUF3130 family. Following the toxin and immunity gene are a cluster of five genes encoding proteins of between 136 and 151 amino acids. The final four genes in this cluster form a module (61-64) that is also found in variable region 1 of some ess C5 and essC7 strains (Figs 5c and S2). The first two genes of the module are also found in some essC3 strains (Fig. 4).

The majority of ess $C 7$ strains have a relatively short variable region 1, comprising genes encoding two small proteins (115 and 113 amino acids, respectively) alongside the toxin- and immunity-encoding genes (Fig. 5c). A few strains also have the ess $\mathrm{C} 6$ four gene module at this region (Fig. $5 \mathrm{c}$ ).

\section{Some ess $C$ variant 1 strains encode truncated EssC2, EssC 3 and EssC4 variants}

During our analysis we observed that some essC1 strains encode the conserved sets of genes found in variable region 1 of other ess $C$ variant strains. For example, strain N11-1255 encodes the complete eight-gene module [21-28] found in ess C2 strains, strain FSL L7-0763 encodes the candidate toxin found in essC3 strains and strain CFSAN026581 encodes the full seven-gene module from ess $\mathrm{C} 4$ strains (Fig. 6a). In each of these cases, a truncated Ess $\mathrm{C}$ protein comprising the C-terminal $\sim 728$ amino acids is also encoded immediately preceding these 'foreign' genes. The truncated EssCs (which are annotated as pseudogenes on the FlaGs output in Fig. S2) are much shorter than the full-length EssC1 protein encoded by these strains; each one aligns to the canonical EssC sequence at residue 771 (Fig. 1d) and has a potential initiator methionine a few codons away. Strikingly, these truncated EssCs do not have the EssC1 variable region, instead the variable region is from the same ess $C$ variant as the foreign genes they harbour (i.e. ess C2 for N11-1255, essC3 for FSL L7-0763 and essC4 for CFSAN026581).

We also noted that some essC1 strains only encode part of the essC2 and ess C4 conserved modules (Fig. 6b). For example, strain PNUSAL000361 harbours genes 24-28 of the ess $C 2$ module but lacks genes 21-23, and strain 25B09 encodes the final four genes [44-47] of the ess C4 conserved cluster, including the shorter version of gene 44 (Fig. S2). In these instances, no truncated EssC is encoded at the gene cluster. These findings suggest that the first three genes at these conserved clusters may be involved in mediating interaction with the variable region of the cognate EssC, potentially to target substrates or to promote functional assembly of the secretion system.

\section{An LXG toxin is encoded at variable region 2 of many strains}

The second variable region lies between the tRNA gene lmot01 and lmo0082, which encodes a small membrane protein of unknown function (Fig. 3a). We noted that while approximately $25 \%$ of strains have no 'insertion' in this region, many other strains have a small locus of two or three genes relating to the T7SS (Figs 7 and S2). Commonly the locus comprises a gene encoding an LXG toxin, along with one or two genes encoding probable immunity proteins (Fig. 7). Four different toxins were identified in variable region 2 across strains (Table 3; toxins D-G; Fig. S5). All four toxins have a highly conserved $\mathrm{N}$-terminal region - the first 330 or so amino acids are almost invariant, but the sequences diverge substantially in the toxin domain (Fig. S5).

A small number of strains (approximately $4 \%$ of those we analysed) have two genes at this locus encoding a protein of 262 amino acids with three predicted transmembrane domains at its N-terminus and a one of 207 amino acids with an N-terminal Tir domain (Fig. S2). We also observed that approximately $5 \%$ of strains had undergone a recombination event next to the tRNA gene to introduce a recombinase gene at this locus (Fig. S2).

\section{LXG proteins are encoded at other loci on the L. monocytogenes chromosome}

To determine whether there may be LXG proteins encoded outside of the L. monocytogenes T7 gene cluster, we selected 14 strains ( 2 of each ess $C$ variant) from those listed in Fig. S2 and searched the genome annotations for the term 'LXG' This yielded 44 protein sequences containing this term, 17 of which we had not previously identified. We next entered the multiple protein accessions collectively into a single round of analysis using the FlaGs tool to see whether any of the encoding genes shared a common genomic location. The output (shown in Fig. S6 and summarized in Table 3) indicates that there are common genomic regions where toxins are likely to be encoded.

To ensure we had covered the diversity of LXG proteins in L. monocytogenes, we next searched RefSeq under the term 'Listeria monocytogenes LXG protein' which returned 569 protein accession numbers. Following manual inspection to see whether they differed from previously identified toxins, we found a further 10 unique protein sequences. 
(a)
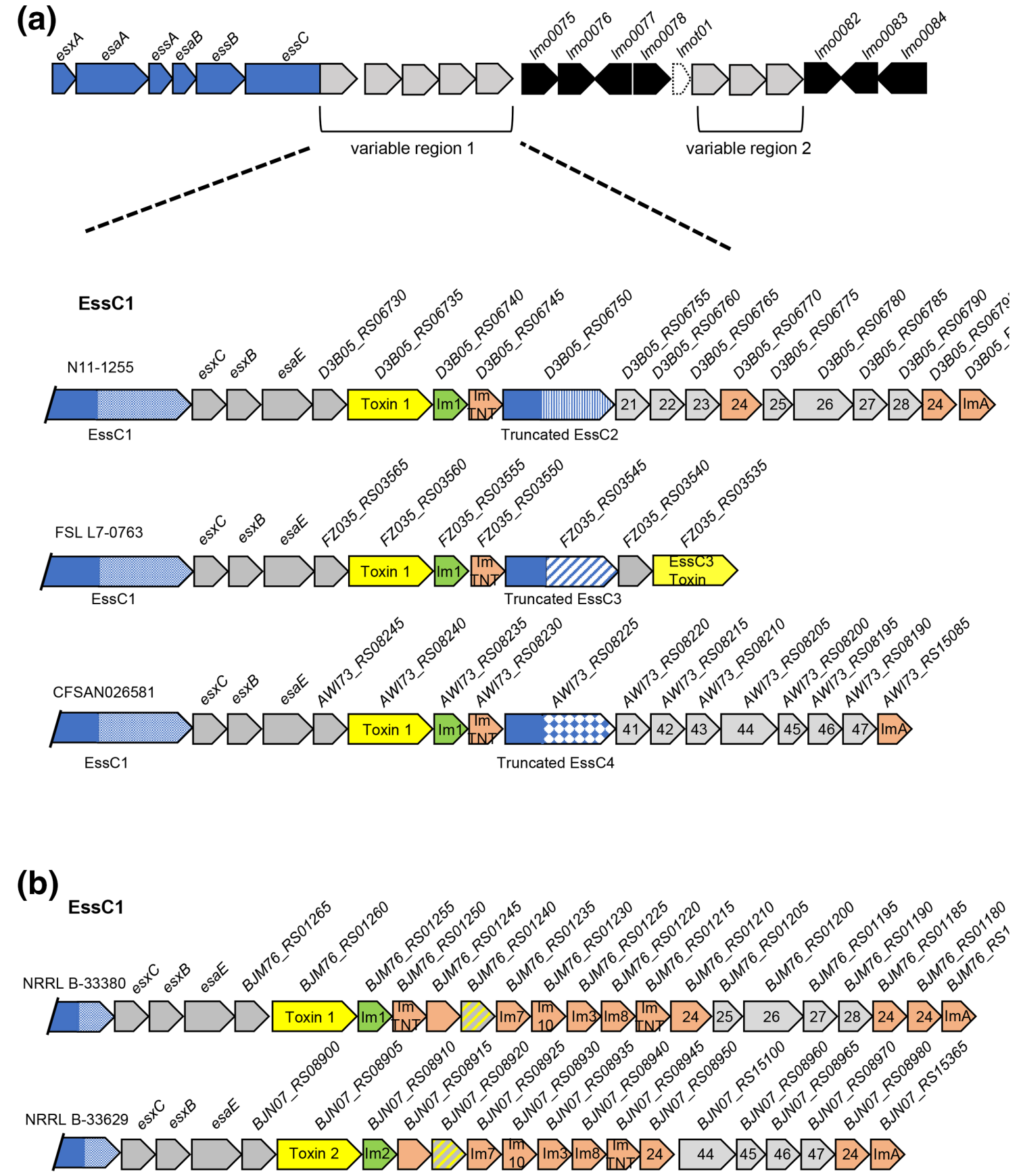

Fig. 6. An additional truncated EssC protein is encoded in some essC1 strains. (a) Genetic arrangement of some example essC1 strains encoding truncated EssCs derived from essC2, essC3 and essC4 strains as indicated. (b) Genetic arrangement of some example essC1 strains that harbour conserved T7 genes from essC2 and essC 4 strains but no truncated EssC. (b) Genes encoding toxins are shaded yellow with the cognate immunity gene in green. Probable orphan immunity proteins are shown in peach (with likely immunity indicated where identified) and genes encoding toxin fragments in hatched grey and yellow shading. Genes numbered 21-28 are common across all essC2 strains; genes numbered 41-47 are common across all essC4 strains. Gene sizes and intergenic regions not to scale.

To identify the chromosomal loci where these additional LXG proteins were encoded, we used two accessions for each toxin that we identified that is encoded outside of the T7 locus and collectively submitted them for flanking gene analysis (shown in Fig. S7 and summarized in Table 3).
It is clear that there are preferred chromosomal locations where LXG proteins are encoded, shown on a map in Fig. 8. We noted that a cluster of $A B C$ transporter genes (lmo0135/lmo0152) is a hotspot for LXG protein-encoding genes, and across $L$. monocytogenes strains we detected 

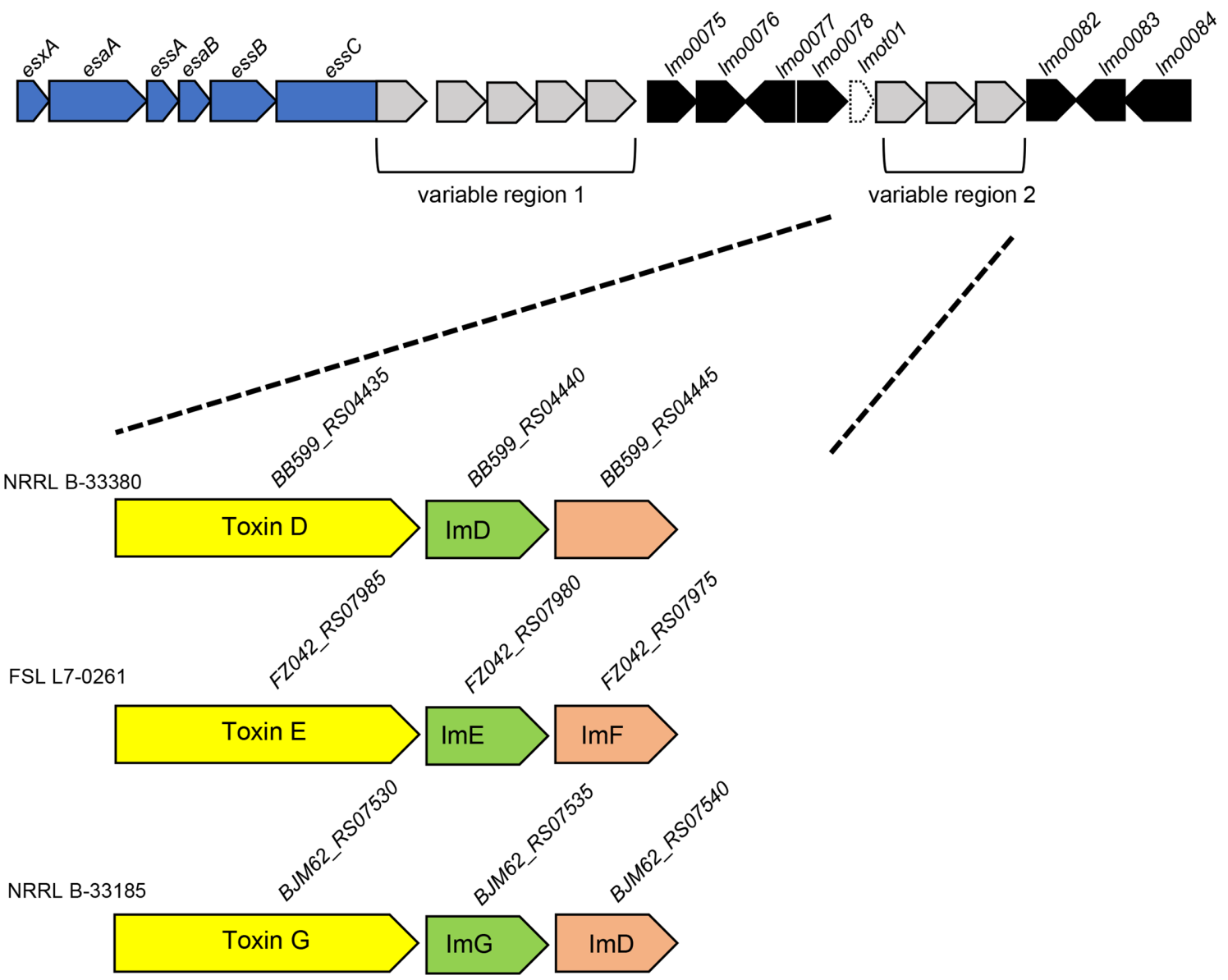

Fig. 7. LXG toxins are often encoded in variable region 2. Three genetic arrangements of variable region 2. Genes encoding candidate toxins are shaded yellow with the cognate immunity gene in green. Probable orphan immunity proteins are shown in peach (with likely immunity indicated where identified). Gene sizes and intergenic regions not to scale.

11 different candidate toxins (toxins $\mathrm{H}-\mathrm{R}$ in Table 3) encoded at that region. Seven unique LXG proteinencoding sequences were also identified at an insertion site between DNA-directed RNA polymerase- and transketolase (lmo0334/lmo0342)-encoding genes (toxins S-Y in Table 3). As far as we could determine, each individual LXG protein is encoded at the same chromosomal locus across the strains. Other hotspots were also identified, although these generally encode fewer unique sequences.

The loci share a number of common features; insertions generally encode a single LXG protein, with the exception of the insertion between the glutamine-hydrolysing GMP synthase and $\alpha / \beta$ hydrolase-encoding genes, where two LXG protein-encoding genes are arranged in a headto-head organization (Fig. S7). Also encoded at the LXG loci are the likely cognate immunity proteins and often additional orphan immunities and toxin fragments. Interestingly, in almost all instances the LXG-encoding gene is preceded by a pair of genes encoding the same two small proteins (numbered 4 and 3 in Figs S6 and 1 and 2 in Fig. S7). Although neither of these is classified as being in the WXG100 superfamily, structural prediction suggests that they share the same WXG100 protein fold, and gene number 4 in Fig. S6/2 in Fig. S7 encodes a protein of the DUF3130/SACOL2603 family that has been linked to the T7SS by phylogenetic profiling.

It is striking to note that all of the LXG proteins encoded next to this gene pair share a similar LXG domain sequence (Fig. S8), although proteins encoded at the same loci are clearly more closely related. Whitney et al. [30] have previously shown that WXG proteins bind to their cognate LXG proteins to promote export by the T7SS. It is very likely that one or both of these small proteins plays a similar role, presumably interacting with the highly conserved $\mathrm{N}$-terminal region. We also observed that three LXG proteins, toxins $\theta, \mu$ and $\phi$, are quite divergent in their $\mathrm{N}$-terminal sequence (Fig. S8). In the case of toxins $\theta$ and $\phi$, they are preceded by a different pair of small genes (346/349 and 319/324, respectively, in Fig. S7) that may encode cognate export partners. No candidate small genes immediately precede toxins $\sigma$ and $\mu$, and they therefore may rely on partner proteins encoded elsewhere in the genome. 


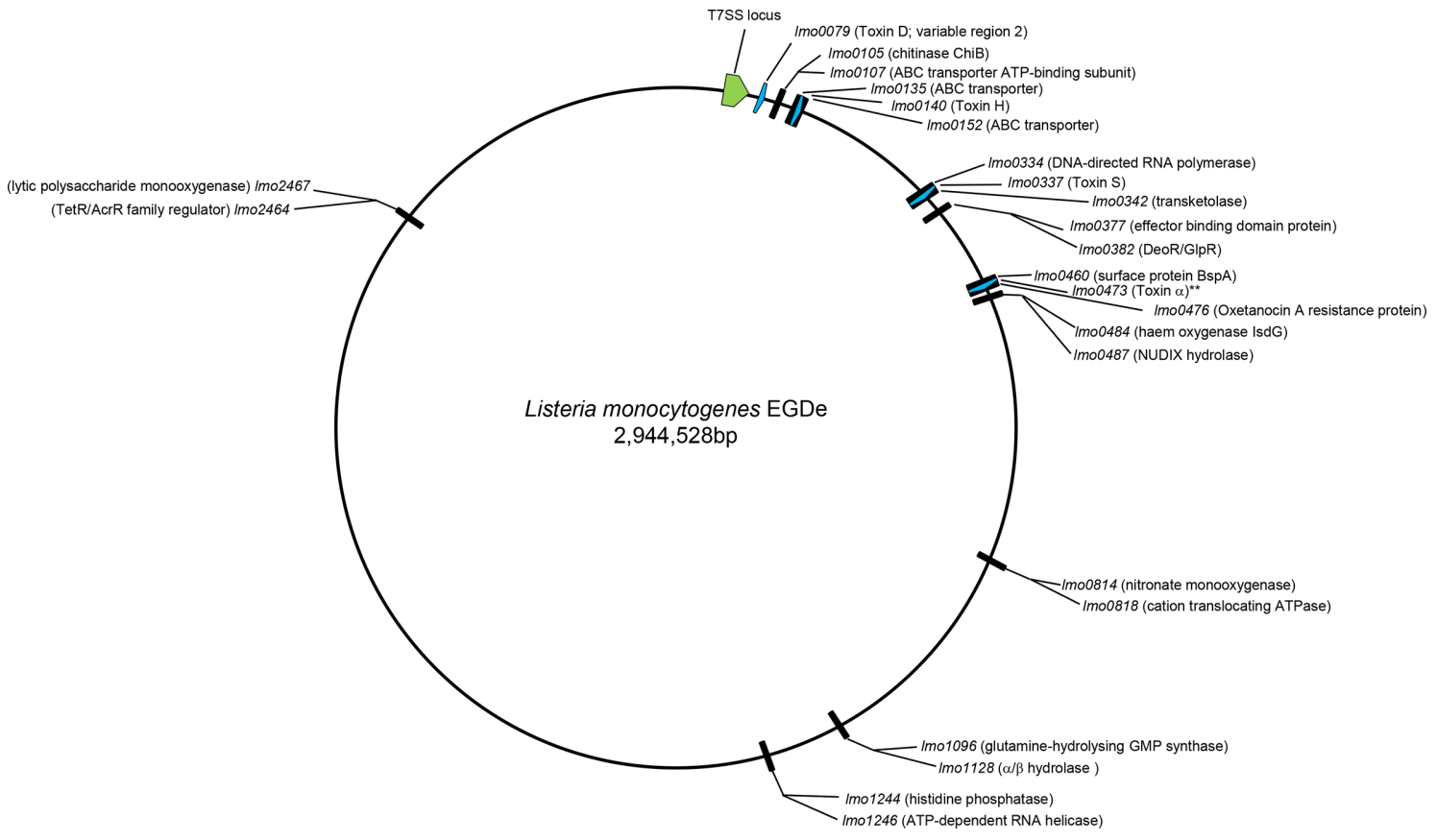

Fig. 8. Chromosomal positions of LXG protein-encoding genes in L. monocytogenes. The positions of the loci at which $L X G$ proteins may be encoded are shown on a genomic map of strain EGDe. LXG protein encoding in EDGe is shown as blue arrows, with the toxin assignment indicated. ${ }^{* *}$ indicates the truncated variant of toxin $\alpha$. The position of the T7 locus is indicated in green.

Finally, when we analysed the $b \operatorname{spA} /$ oxrB (lmo0460/lmo0476) locus in the genome of EGDe where we had observed LXG protein-encoding genes in other strains, we observed that this strain encodes an unusual truncated LXG domain protein at this locus (Fig. S7). The protein, Lmo0473, lacks the first 130 amino acids of a canonical LXG domain protein and is preceded by a gene encoding a protein with a bacteriophage abortive infection $\mathrm{AbiH}$ domain. A second $\mathrm{AbiH}$ domain protein is also encoded nearby, and it appears that EGDe and a few other strains may have undergone a genomic rearrangement or insertion event at the locus. A manual search using the sequence of Lmo0473 revealed that several L. monocytogenes strains encode an otherwise sequence identical protein at this locus, but it is longer and is a bona fide LXG protein (toxin $a$ on the sequence alignment shown in Fig. S8). Curiously, these longer proteins are not found in the RefSeq database, which is used as input for the FlaGs program (and are therefore missing from Fig. S7), although there are several entries in the NCBI's non-redundant protein sequence database. It is not clear whether the truncated variant encoded by EGDe and other strains would be a substrate for the T7SS or if it may use an alternative secretion pathway.

\section{DISCUSSION}

In this study we have undertaken a genomic survey of the T7SS of L. monocytogenes. Our results show that there is exceptional diversity across strains in both the identity of the core component EssC and the likely secreted substrates.
We identified seven variants of EssC based on the sequence of the C-terminal 500-600 amino acids. The most common variant is EssC1, which is encoded in just over a quarter of sequenced L. monocytogenes strains, including the reference strain EGDe. The genetic organization of ess $\mathrm{C} 1$ to some extent mirrors that of $S$. aureus ess $\mathrm{C} 1$ strains, with genes encoding the secreted substrates EsxC and EsxB and the chaperone protein EsaE common between the two, and sharing conserved positioning. A toxin with an $\mathrm{N}$-terminal YeeF domain is found in all L. monocytogenes ess $C 1$ strains. In S. aureus EsaE interacts with the YeeF domain of $\mathrm{EsaD}$ and with $\mathrm{EssC} 1$ and appears to target the toxin to the secretion machinery $[34,36]$. It is likely that the YeeF domain toxins are similarly targeted in L. monocytogenes. Since we failed to identify an EsaElike protein encoded in any of the other ess $C$ variants, we speculate that other substrate proteins, such as the LXG domain proteins, do not require an EsaE-like protein for targeting to the T7SS.

Strains encoding the EssC5, EssC6 and EssC7 variants are the least common. These strains share some similarity at the ess $C$ locus; all three strains encode an LXG domain protein and likely immunity protein downstream of ess $C$. In each case the LXG protein-encoding gene is separated from ess C by two intervening genes. Based on the positioning of these genes and the fact that one or both encode small proteins, we speculate that they may be LXG partners required to mediate export. 
For the other three ess $C$ variants the identity of substrate protein(s) encoded immediately downstream of ess $C$ is not obvious. Strains harbouring essC3 variants always encode the same two proteins downstream, the larger of which may represent a novel secretion substrate. The essC2 and essC4 variants have clusters of genes in the vicinity of ess $C$ that mainly encode proteins of unknown function. Some or all of these genes are frequently found at the ess $C$ locus of other variant strains. Often a smaller module of five genes (essC2) or four genes (essC4) is found across other strain variants, including some ess $\mathrm{C} 1$ strains. Curiously, however, some other ess $\mathrm{C} 1$ strains contain the full complement of 'foreign' genes - in this case the strains always adjacently encode an 'orphan' truncated EssC covering the C-terminal 728 amino acids, which shares the same sequence as the ess $C$ variant from where the genes were acquired. It is not clear whether these shorter EssC proteins (which lack transmembrane domains) are functional and can integrate into the T7SS. Alternatively, they may serve as a site of recombination with the full-length ess $C$ gene, which would splice out the intervening genes and convert an ess $\mathrm{C} 1$ variant into an essC2/essC4 strain.

We identified a second variable locus at the T7 gene cluster that is present in most, but not all, strains. One of four different proteins, each sharing a highly conserved LXG domain, was found to be encoded at this region. Outside of the T7 cluster we also identified additional loci where LXG proteins are encoded. In total, across all strains we identified 40 different LXG proteins. Although none of these LXG proteins have yet been characterized, some of them could be structurally modelled on nuclease, $\mathrm{NAD}^{+}$ glycohydrolase and membrane-depolarising toxins. Taken together, our findings imply an important role for the T7SS secretion system in bacterial antagonism and kin discrimination in L. monocytogenes. We note that prior studies failed to demonstrate a function for the T7SS in virulence and host cell invasion, if anything finding that it had a negative impact on L. monocytogenes infection. Intriguingly, genes encoding some of the T7SS components and the YeeF domain toxin are upregulated when strain EGDe is used to infect germ-free mice that have been colonized with Lactobacillus [59], which would be consistent with a role in interspecies competition. Our findings provide a framework for the functional analysis of the T7SS across the species.

\section{Funding information}

This study was supported by the Wellcome Trust (through Investigator Award 10183/Z/15/Z to T. P.).

\section{Acknowledgements}

We are grateful to Stephen Garrett (Newcastle University), Chayan Kumar Saha and Dr Gemma Atkinson (Umea University) for helpful advice with the FlaGs programmme. We thank Dr Felicity Alcock, Dr Lisa Bowman and Stephen Garrett for critical reading of the manuscript.

Conflicts of interest

The authors declare that there are no conflicts of interest.
References

1. Costa TRD, Felisberto-Rodrigues C, Meir A, Prevost MS, Redzej A et al. Secretion systems in gram-negative bacteria: structural and mechanistic insights. Nat Rev Microbiol 2015;13:343-359.

2. Gorasia DG, Veith PD, Reynolds EC. The type IX secretion system: advances in structure, function and organisation. Microorganisms 2020;8:1173.

3. Palmer T, Finney AJ, Saha CK, Atkinson GC, Sargent F. A holin/ peptidoglycan hydrolase-dependent protein secretion system. Mol Microbiol 2021:in press.

4. Bunduc CM, Bitter W, Houben ENG. Structure and function of the mycobacterial type VII secretion systems. Annu Rev Microbiol 2020;74:315-335

5. Klein TA, Ahmad S, Whitney JC. Contact-dependent interbacterial antagonism mediated by protein secretion machines. Trends Microbiol 2020;28:387-400.

6. Hsu T, Hingley-Wilson SM, Chen B, Chen M, Dai AZ et al. The primary mechanism of attenuation of Bacillus Calmette-Guerin is a loss of secreted lytic function required for invasion of lung interstitial tissue. Proc Natl Acad Sci U S A 2003;100:12420-12425.

7. Pym AS, Brodin P, Majlessi L, Brosch R, Demangel C et al. Recombinant BCG exporting ESAT-6 confers enhanced protection against tuberculosis. Nat Med 2003;9:533-539.

8. Stanley SA, Raghavan S, Hwang WW, Cox JS. Acute infection and macrophage subversion by Mycobacterium tuberculosis require a specialized secretion system. Proc Natl Acad Sci U S A 2003;100:13001-13006.

9. Burts ML, Williams WA, DeBord K, Missiakas DM. EsxA and EsxB are secreted by an ESAT-6-like system that is required for the pathogenesis of Staphylococcus aureus infections. Proc Natl Acad Sci U S A 2005:102:1169-1174.

10. Burts ML, DeDent AC, Missiakas DM. EsaC substrate for the ESAT-6 secretion pathway and its role in persistent infections of Staphylococcus aureus. Mol Microbiol 2008;69:736-746.

11. Abdallah AM, Gey van Pittius NC, Champion PAD, Cox J, Luirink J et al. Type VII secretion--mycobacteria show the way. Nat Rev Microbiol 2007:5:883-891.

12. Unnikrishnan M, Constantinidou C, Palmer T, Pallen MJ. The enigmatic Esx proteins: looking beyond mycobacteria. Trends Microbiol 2017;25:192-204.

13. Rosenberg OS, Dovala D, Li X, Connolly L, Bendebury A et al. Substrates control multimerization and activation of the multidomain ATPase motor of type VII secretion. Cell 2015;161:501-512.

14. Zoltner M, Ng WMAV, Money JJ, Fyfe PK, Kneuper $\mathrm{H}$ et al. EssC: domain structures inform on the elusive translocation channel in the Type VII secretion system. Biochem J 2016:473:1941-1952.

15. Beckham KSH, Ciccarelli L, Bunduc CM, Mertens HDT, Ummels R et al. Structure of the mycobacterial ESX-5 type VII secretion system membrane complex by single-particle analysis. Nat Microbiol 2017:2:17047.

16. Famelis N, Rivera-Calzada A, Degliesposti $G$, Wingender $M$, Mietrach $\mathrm{N}$ et al. Architecture of the mycobacterial type VII secretion system. Nature 2019;576:321-325.

17. Poweleit N, Czudnochowski N, Nakagawa R, Trinidad DD, Murphy KC et al. The structure of the endogenous ESX-3 secretion system. Elife 2019:8:e52983.

18. Ramsdell TL, Huppert LA, Sysoeva TA, Fortune SM, Burton BM. Linked domain architectures allow for specialization of function in the FtsK/SpollIE ATPases of Esx secretion systems. J Mol Biol 2015;427:1119-1132.

19. Mietrach N, Damián-Aparicio D, Mielich-Süss B, Lopez D, Geibel S. Substrate interaction with the EssC coupling protein of the type VIIB secretion system. J Bacteriol 2020;202:e00646-19.

20. Wang S, Zhou K, Yang X, Zhang B, Zhao Y et al. Structural insights into substrate recognition by the type VII secretion system. Protein Cell 2020;11:124-137. 
21. Jäger $F$, Kneuper $H$, Palmer T. EssC is a specificity determinant for Staphylococcus aureus type VII secretion. Microbiology 2018;164:816-820.

22. Champion PAD, Stanley SA, Champion MM, Brown EJ, Cox JS. C-terminal signal sequence promotes virulence factor secretion in Mycobacterium tuberculosis. Science 2006;313:1632-1636.

23. Anderson M, Aly KA, Chen Y-H, Missiakas D. Secretion of atypical protein substrates by the ESAT-6 secretion system of Staphylococcus aureus. Mol Microbiol 2013;90:734-743.

24. Sysoeva TA, Zepeda-Rivera MA, Huppert LA, Burton BM. Dimer recognition and secretion by the ESX secretion system in Bacillus subtilis. Proc Natl Acad Sci U S A 2014;111:7653-7658.

25. Kneuper H, Cao ZP, Twomey KB, Zoltner M, Jäger F et al. Heterogeneity in ess transcriptional organization and variable contribution of the Ess/Type VII protein secretion system to virulence across closely related Staphylocccus aureus strains. Mol Microbiol 2014:93:928-943.

26. Jäger $F$, Zoltner M, Kneuper H, Hunter WN, Palmer T. Membrane interactions and self-association of components of the Ess/ Type VII secretion system of Staphylococcus aureus. FEBS Lett 2016:590:349-357.

27. Casabona MG, Buchanan G, Zoltner $M$, Harkins CP, Holden MTG et al. Functional analysis of the EsaB component of the Staphylococcus aureus Type VII secretion system. Microbiology 2017;163:1851-1863.

28. Huppert LA, Ramsdell TL, Chase MR, Sarracino DA, Fortune SM et al. The ESX system in Bacillus subtilis mediates protein secretion. PLoS One 2014:9:e96267.

29. Baptista C, Barreto HC, São-José C. High levels of DegU-P activate an Esat-6-like secretion system in Bacillus subtilis. PLoS One 2013;8:e67840.

30. Whitney JC, Peterson SB, Kim J, Pazos M, Verster AJ et al. A broadly distributed toxin family mediates contact-dependent antagonism between gram-positive bacteria. Elife 2017;6:e26938.

31. Dai $Y$, Wang $Y$, Liu Q, Gao Q, Lu H et al. A Novel ESAT-6 secretion system-secreted protein EsxX of community-associated Staphylococcus aureus lineage ST398 contributes to immune evasion and virulence. Front Microbiol 2017:8:819.

32. Way SS, Wilson CB. The Mycobacterium tuberculosis ESAT-6 homologue in Listeria monocytogenes is dispensable for growth in vitro and in vivo. Infect Immun 2005;73:6151-6153.

33. Warne B, Harkins CP, Harris SR, Vatsiou A, Stanley-Wall N et al. The Ess/Type VII secretion system of Staphylococcus aureus shows unexpected genetic diversity. BMC Genomics 2016;17:222.

34. Cao Z, Casabona MG, Kneuper H, Chalmers JD, Palmer T. The type VII secretion system of Staphylococcus aureus secretes a nuclease toxin that targets competitor bacteria. Nat Microbiol 2016;2:16183.

35. Ohr RJ, Anderson M, Shi M, Schneewind O, Missiakas D. EssD, a nuclease effector of the Staphylococcus aureus ESS pathway. $J$ Bacteriol 2017;199:e00528-16.

36. Anderson M, Ohr RJ, Aly KA, Nocadello S, Kim HK et al. EssE Promotes Staphylococcus aureus ESS-dependent protein secretion to modify host immune responses during infection. J Bacteriol 2017:199:e00527-16.

37. Ulhuq FR, Gomes MC, Duggan GM, Guo M, Mendonca C et al. A membrane-depolarizing toxin substrate of the Staphylococcus aureus type VII secretion system mediates intraspecies competition. Proc Natl Acad Sci U S A 2020;117:20836-20847.

38. Chatterjee A, Willett JLE, Dunny GM, Duerkop BA. Phage infection and sub-lethal antibiotic exposure mediate Enterococcus faecalis type VII secretion system dependent inhibition of bystander bacteria. PLoS Genet 2021;17:e1009204.

39. Tassinari M, Doan T, Bellinzoni M, Chabalier M, Ben-Assaya M. Central role and structure of the membrane pseudokinase YukC in the antibacterial Bacillus subtilis type VIIb secretion system. bioRxiv 2020:05.09.085852.
40. Radoshevich L, Cossart P. Listeria monocytogenes: towards a complete picture of its physiology and pathogenesis. Nat Rev Microbiol 2018;16:32-46.

41. Pinheiro J, Reis O, Vieira A, Moura IM, Zanolli Moreno L et al. Listeria monocytogenes encodes a functional ESX-1 secretion system whose expression is detrimental to in vivo infection. Virulence 2017:8:993-1004.

42. Altschul SF, Madden TL, Schäffer AA, Zhang J, Zhang Z et al. Gapped BLAST and PSI-BLAST: a new generation of protein database search programs. Nucleic Acids Res 1997;25:3389-3402.

43. Madeira F, Park YM, Lee J, Buso N, Gur T et al. The EMBL-EBI search and sequence analysis tools Apis in 2019. Nucleic Acids Res 2019:47:W636-W641

44. Waterhouse AM, Procter JB, Martin DMA, Clamp M, Barton GJ. Jalview Version 2-a multiple sequence alignment editor and analysis workbench. Bioinformatics 2009:25:1189-1191.

45. Kumar S, Stecher G, Li M, Knyaz C, Tamura K. MEGA X: molecular evolutionary genetics analysis across computing platforms. Mol Biol Evol 2018;35:1547-1549.

46. Stecher G, Tamura K, Kumar S. Molecular evolutionary genetics analysis (MEGA) for macOS. Mol Biol Evol 2020;37:1237-1239.

47. Bécavin C, Koutero M, Tchitchek N, Cerutti F, Lechat P et al. Listeriomics: an interactive web platform for systems biology of Listeria. mSystems 2017;2:e00186-16.

48. Solovyev V, Salamov A. Automatic annotation of microbial genomes and metagenomic sequences. In: RW Li (editor). Metagenomics and its Applications in Agriculture, Biomedicine and Environmental Studies. Nova Science Publishers; 2011. pp. 61-78.

49. Macke TJ, Ecker DJ, Gutell RR, Gautheret D, Case DA et al. RNAMotif, an RNA secondary structure definition and search algorithm. Nucleic Acids Res 2001:29:4724-4735.

50. Saha CK, Pires RS, Brolin H, Delannoy M, Atkinson GC. Flags and webFlaGs: discovering novel biology through the analysis of gene neighbourhood conservation. Bioinformatics 2020:in press.

51. Krogh A, Larsson B, von Heijne G, Sonnhammer EL. Predicting transmembrane protein topology with a hidden Markov model: application to complete genomes. J Mol Biol 2001;305:567-580.

52. Kelley LA, Mezulis S, Yates CM, Wass MN, Sternberg MJE. The Phyre2 web portal for protein modeling, prediction and analysis. Nat Protoc 2015;10:845-858.

53. Bobrovskyy M, Willing SE, Schneewind O, Missiakas D. EssH peptidoglycan hydrolase enables Staphylococcus aureus Type VII secretion across the bacterial cell wall envelope. J Bacteriol 2018;200:e00268-18.

54. Cao Z. Characterisation of the Type VII secretion system of Staphylococcus aureus: PhD thesis. University of Dundee 2017.

55. Orsi RH, den Bakker HC, Wiedmann M. Listeria monocytogenes lineages: genomics, evolution, ecology, and phenotypic characteristics. Int J Med Microbiol 2011:301:79-96.

56. Ragon M, Wirth T, Hollandt F, Lavenir R, Lecuit M et al. A new perspective on Listeria monocytogenes evolution. PLOS Pathog 2008;4:e1000146.

57. Kuenne C, Billion A, Mraheil MA, Strittmatter A, Daniel R et al. Reassessment of the Listeria monocytogenes pan-genome reveals dynamic integration hotspots and mobile genetic elements as major components of the accessory genome. BMC Genomics 2013:14:47.

58. Rychli K, Wagner EM, Ciolacu L, Zaiser A, Tasara T et al. Comparative genomics of human and non-human Listeria monocytogenes sequence type 121 strains. PLoS One 2017;12:e0176857.

59. Archambaud C, Nahori M-A, Soubigou G, Bécavin C, Laval L et al. Impact of lactobacilli on orally acquired listeriosis. Proc Natl Acad Sci U S A 2012:109:16684-16689.

Edited by: R. Manganelli and P. Brodin 